\section{Jean-Baptiste Michel Jean-François Arnal}

\section{ADRESSES}

J.B. Michel : directeur de recherche à l'Inserm. J.F. Arnal : interne des Hôpitaux de Paris Inserm U. 367, 17, rue du Fer-à-Moulin, 75005 Paris, France.

\title{
Monoxyde d'azote et hypertension artérielle
}

Le monoxyde d'azote (NO) joue un rôle essentiel dans le maintien d'un tonus vasodilatateur. Ce composé est synthétisé par les NO-synthases dont l'activité, dans les cellules endothéliales, est réglée par la concentration intracellulaire de calcium, elle-même modulée par des substances vasoactives (acétylcholine, bradykinine) et les forces de cisaillement liées au flux sanguin. Le NO agit en stimulant une guanylate cyclase soluble des cellules musculaires lisses de la paroi vasculaire, et son effet est donc relayé par l'augmentation de la concentration intracellulaire de GMP cyclique. La voie de régulation NOGMP cyclique pourrait être perturbée dans certains types d'hypertension artérielle et dans des modifications chroniques du débit sanguin (insuffisance cardiaque, fistule artérioveineuse) ou des propriétés de la paroi vasculaire (vieillissement, athérosclérose...).

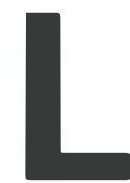

'endothélium joue un rôle crucial à l'interface entre la phase liquide du sang circulant et la paroi artérielle, solide visco-élastique. L'endothélium prévient la coagulation de la phase liquide par la synthèse de différents facteurs, reçoit des informations de cette phase (le sang) et les transmet à la phase solide (la média), modulant son tonus contractile et sa trophicité. La média artérielle est constituée uniquement de cellules musculaires lisses, contractiles, et de matrice extracellulaire. Furchgott et Zawadski [1] furent les premiers à montrer que la relaxation des cellules musculaires lisses artérielles en réponse à l'acétylcholine dépendait de l'intégrité anatomique de l'endothé- lium. Ils baptisèrent le principe de cette relation intercellulaire EDRF (endothelium derived relaxing factor).

\section{Hémodynamique sanguine} et endothélium

L'EDRF fut d'abord caractérisé comme une substance diffusible ayant une demi-vie très brève. Il fallut sept années pour faire la preuve que l'EDRF n'était autre que le monoxyde d'azote (NO) $[2,3]$. Le NO est produit par une famille d'enzymes, les NO-synthases, à partir d'un substrat commun, la Larginine. Trois types de NOsynthases ont été caractérisés : la NO-synthase inductible macrophagique, la NO-synthase constitutive du 


\section{RÉFÉRENCES}

1. Furchgott RF, Zawadski JV. The obligatory role of endothelial cells in the relaxation of arterial smooth muscle by acetylcholine. Nature 1980 ; 28 : 373-6.

2. Ignarro LJ, Buga GM, Wood KS, Byrns RE, Chanduri G. Endothelial derived relaxing factor produced and released from artery and vein is nitric oxyde. Proc Natl Acad Sci USA 1987 ; 84 : 9265-9

3. Moncada S, Palmer RMJ, Higgs EA. Nitric oxide : physiology, pathophysiology and pharmacology. Pharmacol Rev $1991 ; 43$ : 109-42.

4. Rapoport RM, Draznin MB, Murad F Endothelium-dependent relaxation in rat aorta may be mediated through cyclic GMPdependent protein phosphorylation. Nature 1983 ; 306 : 174-6.

5. Rees DD, Palmer RM J, Moncada S. Role of endothelium derived nitric oxyde in the regulation of blood pressure. Proc Natl Acad Sci USA 1989 ; 86 : 3375-8

6. Davies PF, Tripathi SC. Mechanical stress mechanisms and the cell. An endothelial paradigm. Circ Res 1993 ; 72 : 239-45.

7. Pohl U, Holtz T, Busse R, Bassenge E. Crucial role of endothelium in the vasodilatator response to increased flow in vivo. Hypertension $1986 ; 8: 37-47$

8. Griffith TM, Edwards DH, Davies R Li, Harrison TJ, Evans KT. EDRF coordinates the behaviour of vascular resistance vessels. Nature 1987 ; 329 : 442-5.

9. Languille BL, O'Donnell F. Reduction in arterial diameter produced by chronic decreases in blood flow are endothelium-dependent. Science $1986 ; 231$ : 405-7.

10. Languille BL., Bendeck MP, Kecley F. Adaptation of carotid arteries of young and mature rabbits to reduced carotid blood flow. Am J Physiol 1989 ; 256 : H931-9.

11. Tolins JP, Palmer RMJ, Moncada S, Raij L. Role of endothelium derived relaxing factor in regulation of renal hemodynamic responses. Am J Physiol 1990 ; 258 : H655-62. 12. Arnal JF, Warin L, Michel JB. Determinants of aortic cyclic GMP in hypertension induced by chronic inhibition of nitric oxyde synthase. J Clin Invest 1992 ; 90 : 647-52.

13. Bayliss C, Mitruka B, Deng A. Chronic blockade of nitric oxyde synthesis in the rat produces systemic hypertension and glomerular damage. J Clin Invest 1992 ; 90 : 278-81. 14. Ribeiro MO, Antunes E, de Nucci G, Lovisolo SM, Zatz R. Chronic inhibition of nitric oxyde synthesis. A new model of arterial hypertension. Hypertension 1992 ; 20 : 298-303

15. Arnal JF, El-Amrani AIK, Chatellier G, Ménard J, Michel JB. Cardiac weight in hypertension induced by nitric oxide-synthase blockade. Hypertension 1993 (sous presse).

16. Gardes J, Poux JM, Gonzalez MF, Alhenc-Gelas F, Menard J. Decreased renin release and constant kallicrein secretion after in jection of L-NAME in isolated perfused rat kidney. Life Sci 1992 ; 50 : 987-93.

17. Van de Voorde J, Lensen I. Endothelium-dependent and independent relaxation of aortic rings from hypertensive système nerveux central et la NOsynthase constitutive spécifique de l'endothélium vasculaire. Au niveau de la paroi vasculaire et dans des conditions physiologiques, la NOsynthase constitutive est présente dans l'endothélium. Il existe également des terminaisons nitroxidergiques dans l'adventice de certains territoires artériels. La NO-synthase inductible peut apparaître dans les cellules vasculaires en réponse à des stimuli pathologiques tels que des endotoxines bactériennes au cours du choc septique. Le NO produit par les cellules endothéliales diffuse et active la guanylate cyclase soluble des cellules musculaires lisses sous-jacentes. Le GMP cyclique produit, second messager du NO, entraîne une cascade de phosphorylation des protéines impliquées dans le recaptage et l'extrusion du calcium, induisant la relaxation de la cellule musculaire lisse, et, in vivo, la vasodilatation [4]. Les différents éléments de la cascade biochimique de la NO-synthase et de la production de $\mathrm{NO}$ ont déjà été présentés dans ce journal $(\mathrm{m} / \mathrm{s}$ n० 10 , vol. 7 , p. 1094-1095, $\mathrm{m} / \mathrm{s} \quad n^{\circ} 8$, vol. 8 , p. 843-845).

La synthèse d'antagonistes compétitifs de la L-arginine par le groupe de Moncada a permis d'étudier l'effet du blocage de la NO-synthase. L'application de ces antagonistes sur des préparations d'anneaux artériels provoque une augmentation du tonus développé par le vaisseau. Moncada et al. ont démontré qu'il existe en permanence une sécrétion de NO responsable d'un tonus vasodilatateur dépendant de l'endothélium, puisque l'effet de ces antagonistes disparaît après ablation de l'endothélium du vaisseau. In vivo, l'administration aiguë de ces antagonistes s'accompagne d'une élévation de la pression artérielle [5] et d'une baisse des débits régionaux. Ces travaux ont donc montré que le tonus vasodilatateur est permanent, non seulement in vitro mais aussi in vivo. La régulation de la pression artérielle et du débit sanguin apparaît donc comme la résultante de deux tonus, l'un vasodilatateur et l'autre vasoconstricteur, alors que seul ce dernier avait été décrit en physiologie cardiovasculaire jusqu'alors.

Une des particularités du système cardiovasculaire réside dans la capacité qu'ont les contraintes hémodynamiques, donc physiques, de transmettre des signaux à la paroi artérielle [6]. En ce qui concerne le NO, il est apparu rapidement que sa sécrétion de base par l'endothélium vasculaire était sous le contrôle du flux sanguin. L'accélération du flux entraîne une augmentation des forces de frottement sur la couche cellulaire d'interface (endothélium) avec la phase solide (paroi artérielle). Ces forces de frottement, ou contraintes de cisaillement, sont proportionnelles à la vitesse sanguine et à la viscosité, et inversement proportionnelles au cube du rayon du vaisseau (figure 1). Physiologiquement, l'augmentation des contraintes de cisaillement accroît la mobilisation du calcium libre dans la cellule endothéliale, activant la NO-synthase endothéliale dépendante du calcium. La production accrue de NO entraîne une relaxation des cellules musculaires lisses sous-jacentes et donc une vasodilatation (figure 2). Cette régulation des calibres vasculaires par la vitesse du flux sanguin joue un rôle fondamental dans l'adaptation du réseau artériel à l'augmentation des besoins métaboliques tissulaires $[7,8]$. Lors de l'exercice physique pour les muscles squelettiques, de la digestion pour le tube digestif, ou encore de l'accélération de la fréquence cardiaque pour le cœur, l'augmentation du métabolisme tissulaire induit une dilatation des artérioles très distales. Cet effondrement des résistances vasculaires s'accompagne localement d'une élévation des vitesses circulatoires dans le réseau vasculaire d'amont. L'accélération du flux sanguin augmente les contraintes de cisaillement sur l'endothélium à l'origine d'un signal de vasodilatation; l'augmentation du calibre artériel normalise ces mêmes contraintes de cisaillement. Si le stimulus devient permanent, cette adaptation fonctionnelle transitoire se complète d'une adaptation structurale $[9,10]$.

Outre les contraintes de cisaillement, stimulus principal, des facteurs pharmacologiques stimulent la fonction endothéliale de production du NO. Les plus classiques sont l'acétylcholine, la bradykinine, l'endothéline, la substance $\mathrm{P}$, l'histamine, la vasopressine, et d'autres peptides vasoactifs 


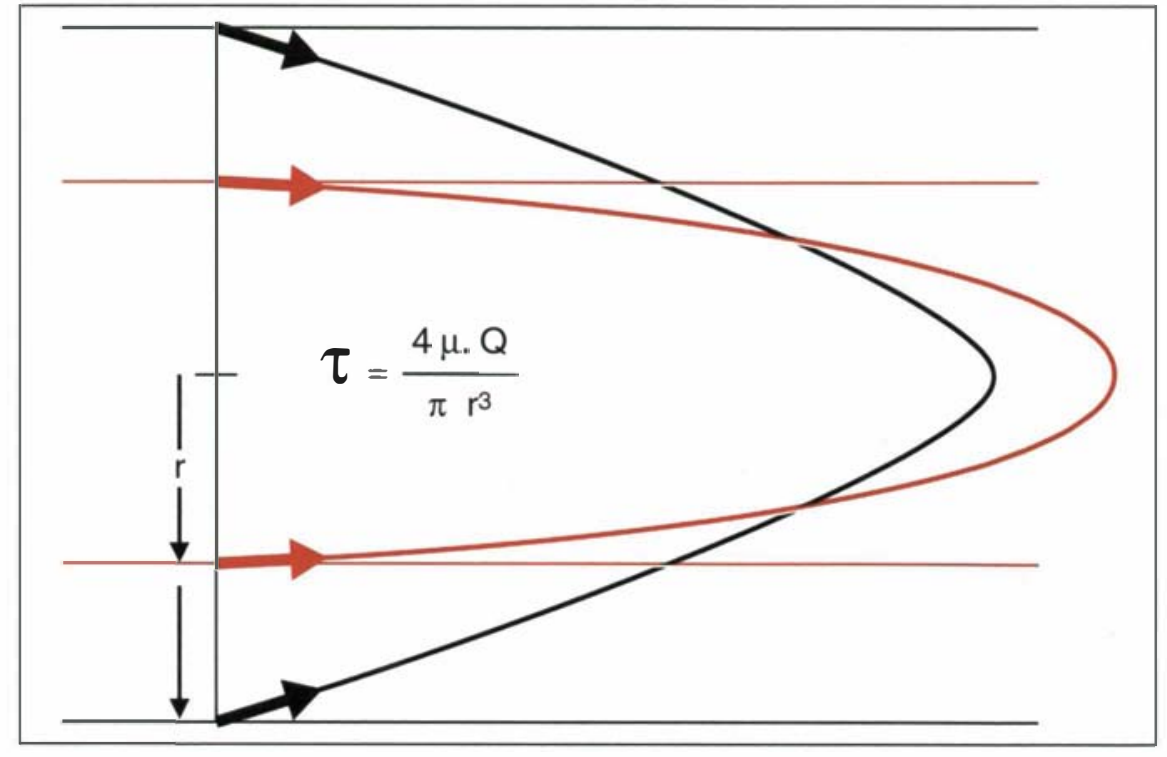

Figure 1. Les contraintes de cisaillements $(\tau)$ de l'écoulement sanguin sur la surface endothéliale $(\rightarrow)$ sont proportionnelles à la viscosité sanguine $(\mu)$, à la vélocité de l'écoulement au niveau de l'interface (Q) et inversement proportionnelles au cube du rayon luminal (r). A l'augmentation de contraintes de cisaillement $(\rightarrow)$ hémodynamiques, l'endothélium répond par une augmentation de sécrétion de NO; la relaxation des cellules musculaires lisses sous-jacentes augmente le diamètre vasculaire qui normalise les contraintes de cisaillement $(\rightarrow)$.

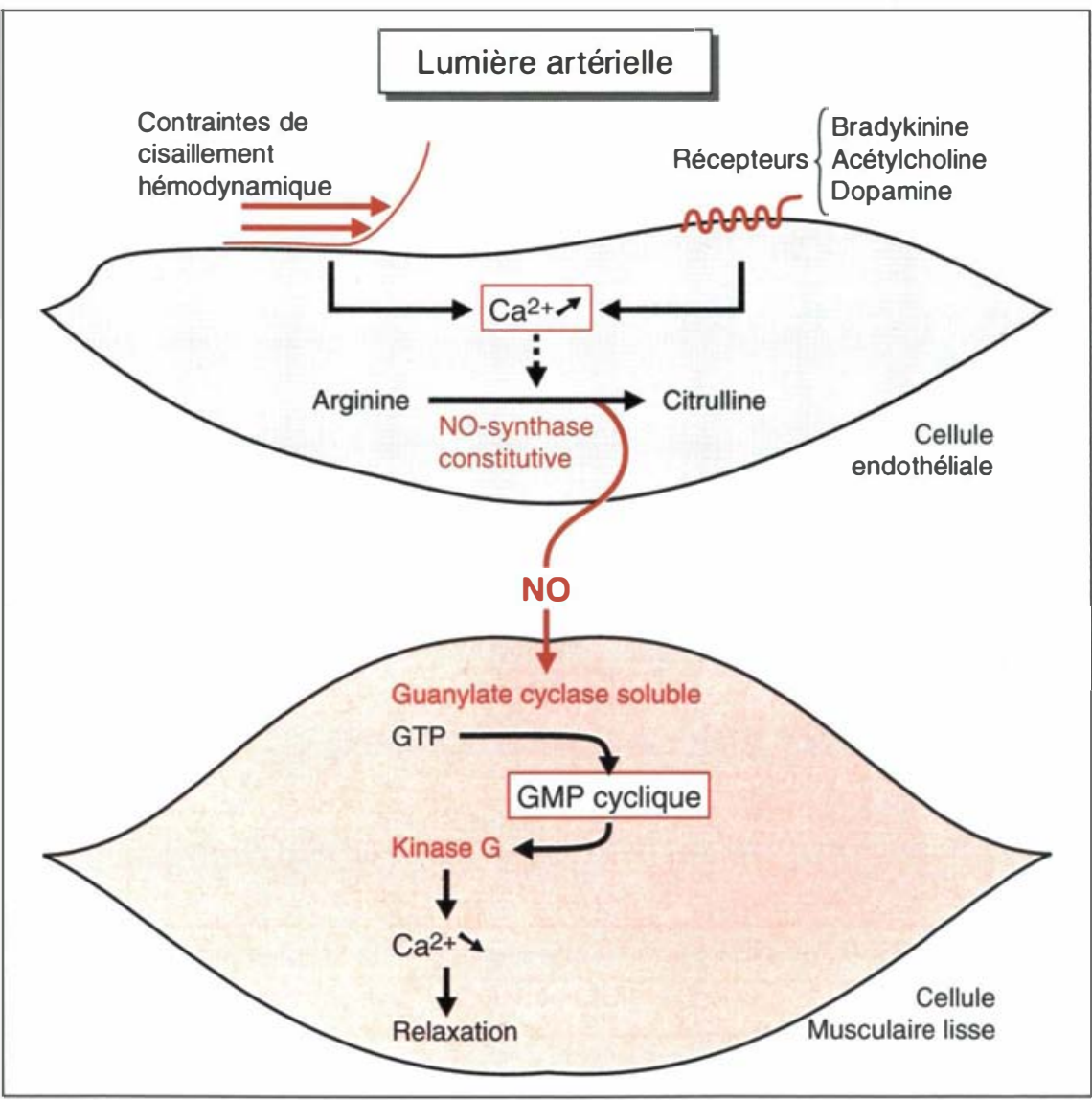

Figure 2. Transduction du signal de la lumière artérielle à la cellule musculaire lisse entraînant sa relaxation.

$m / s n^{\circ} 10$ vol. 9, octobre 93 dont l'effet vasodilatateur dépend de la présence et de l'intégrité fonctionnelle de l'endothélium.

\section{Blocage chronique de la NO-synthase : un nouveau modèle d'hypertension artérielle}

L'injection aiguë d'un antagoniste compétitif de la L-arginine (LNMMA) sur la NO-synthase entraîne une élévation de la pression artérielle [5]. La circulation artérielle rénale est une des cibles privilégiées du blocage aigu de la NOsynthase [11] ; celui-ci provoque une augmentation immédiate des résistances rénales et une diminution de la filtration glomérulaire. Aux expériences de blocage aigu de la NOsynthase, ont succédé des expériences de blocage chronique. Elles sont habituellement réalisées par l'administration chronique de nitro-arginine methyl ester (L-NAME), molécule soluble facile à administrer par voie orale chez l'animal. L'administration chronique de L-NAME chez le rat s'accompagne d'une élévation progressive, dépendante de la dose, de la pression artérielle [12]. Elle s'accompagne d'une diminution, également dépendante de la dose, du contenu en GMP cyclique de la paroi artérielle (figure 3). Ces deux phénomènes, élévation de la pression artérielle et baisse du GMP cyclique pariétal, sont inversés par l'administration d'un donneur de NO, (comme le nitroprussiate de sodium qui active la guanylate cyclase soluble), et par de fortes doses d'arginine, tout au moins dans la première phase réversible de cette hypertension. L'administration chronique de LNAME bloque l'ensemble des NOsynthases et n'est donc pas spécifique de la NO-synthase constitutive endothéliale. Le blocage de la production de NO par les terminaisons nerveuses nitroxidergiques, par action directe sur les cellules musculaires lisses ou modulation de l'interaction synaptique, pourrait également jouer un rôle physiopathologique dans ce modèle.

Cette hypertension artérielle chronique présente deux caractéristiques : l'absence d'hypertrophie ventriculaire gauche et l'atteinte rénale [13-15], 


\section{RÉFÉRENCES}

18. Luscher TF, Vanhoutte PM Endothelium-dependent contractions to acetylcholine in the aorta of spontaneously hypertensive rat. Hypertension $1986 ; 8: 344-8$.

19. Angus JA, Dyke AC, Jennings GL, Korner PI, Sudhir K, Ward JE, Wright CE Release of endothelium-derived relaxing factor from resistance arteries in hypertension. Kidney Internat $1992 ; 41$ (suppl 37) : 573-8. 20. Lacolley PJ, Lervis SI, Brody MJ. L-Ng Nitro arginine produces an exaggerated hypertension in anesthetized SHR. Eur J Pharmacol $1991 ; 197: 239-40$.

21. Arnal JF, Battle T, Mćnard J, Michel JB. The vasodilatory effect of endogenous nitric oxide is a major counter regulatory mechanism in the spontaneously hypertensive rat $J$. Hypertens 1993 (sous presse).

22. Bockman CS, Jeffiries WB, Pettinger WA, Abel PW. Enhanced release of endotheliumderived relaxing factor in mineralocorticoid hypertension. Hypertension 1992 ; 20 : 304-13. 23. Shultz PJ, Tolins JP. Adaptation to increased dietary salt intake in the rat. Role of endogenous nitric oxide. J Clin Invest 1993 ; $91: 642-50$.

24. Chen PY, Sanders PW. L-arginine abrogates salt sensitive hypertensions in Dahl/Rapp rats. J Clin Invest $1991 ; 88$ : 1559-67.

25. Panza JA, Quyyumi AA, Brush JE, Epstein SE. Abnormal endothelium-dependent vascular relaxation in patients with essential hypertension. N Engl JMed $1990 ; 323$ : 22-7. 26. Panza JA, Casino PR, Kilcoyne CM, Quyyumi AA. Role of endothelium-derived nitric oxide in the abnormal endotheliumdependent vascular relaxation of patients with essential hypertension. Circulation $1993 ; 87$ : 1468-74.

27. Panza JA, Casino PR, Badar DM, Quyyumi A A. Effect of increased availability of endothelium-derived nitric oxide precursor on endothelium-dependent vascular relaxation in normal subjects and in patients with essential hypertension. Circulation 1993; 87 : 1475-81.

28. Girerd XJ, Hirsch AT, Cooke JP, Dzau VJ, Creager MA. L-arginine augments endothelium-dependent vasodilatation in hypercholesterolemic rabbits. Circ Res 1990 ; 67: 1301-8.

29. Creager MA, Gallagher SH, Girerd XJ, Coleman S, Dzau VJ, Cooke JP. L-arginine improves endothelium-dependent vasodilatation in hypercholesterolemic humans. J Clin Invest $1992 ; 90: 1248-53$.

30. Arnal JF, Philippe M, Laboulandine I, Michel JB. Effect of perindopril in rat cardiac volume overload. Am Heart J 1993 (sous presse).

31. Ontkean M, Gay R, Greenberg B. Diminished endothelium-derived relaxing factor activity in an experimental of chronic heart failure. Circ Res 1991; 69 : 1088-96.

32. Arnal JF, Hoffman F, Michel JB. Discrepancy between plasma and aortic wall cyclic GMP in an experimental model of congestive heart failure. Cardiovasc Res 1993 ; 27 . 1094-100.

33. Moritoki H, Tanioka A, Maeshiba Y Irvanato T, Ishida $\mathrm{Y}$, Araki $\mathrm{H}$. Age-associated decrease in histamine-induced vasodilatation may be due to reduction of cyclic GMP formation. Br J Pharmacol 1988 ; 95 : 1015-22. probablement plus précoce que dans les modèles expérimentaux classiques d'hypertension artérielle, tels que l'hypertension rénovasculaire, l'administration de désoxycorticostérone et de sel (modèle DOCA-sel), ou les modèles murins d'hypertension artérielle génétique. L'absence d'hypertrophie cardiaque pourrait être en rapport avec les diminutions du débit cardiaque et du volume d'éjection systolique observées dans ce modèle. L'atteinte rénale est d'emblée fonctionnelle, avec une diminution de la filtration glomérulaire et du coefficient d'ultrafiltration, et une augmentation majeure des résistances rénales. Cette atteinte fonctionnelle initiale s'accompagne rapidement de lésions structurales d'artériolopathie proliférative et de glomérulosclérose. L'apparition de ces lésions rénales initie la seconde phase de ce modèle au cours de laquelle l'hypertension artérielle semble moins réversible à l'arrêt de l'intoxication par le LNAME. La précocité de cette atteinte rénale pourrait être en rapport avec un effet direct du blocage de la NOsynthase rénale venant se surajouter aux effets de l'hypertension artérielle. Néanmoins, il reste difficile de préciser exactement ce qui revient à l'augmentation de la pression artérielle et ce qui revient aux effets intrinsèques rénaux du blocage de la production de NO.

L'administration de L-NAME, aiguë ou dans le rein isolé perfusé, freine la sécrétion de rénine [16]. Dans la première phase, réversible et sans atteinte structurale rénale, le taux de rénine est légèrement abaissé dans ce modèle d'intoxication chronique par la L-NAME, alors que dans la seconde phase, avec atteinte structurale rénale, la sécrétion de rénine est activée. Les antagonistes de l'angiotensine II préviennent le développement de cette seconde phase [14].

\section{Implication de la voie du NO-GMP cyclique dans les modèles expérimentaux d'hypertension artérielle}

On classe les modèles usuels d'hypertension artérielle en modèles d'hypertensions artérielles primitives, d'ori-

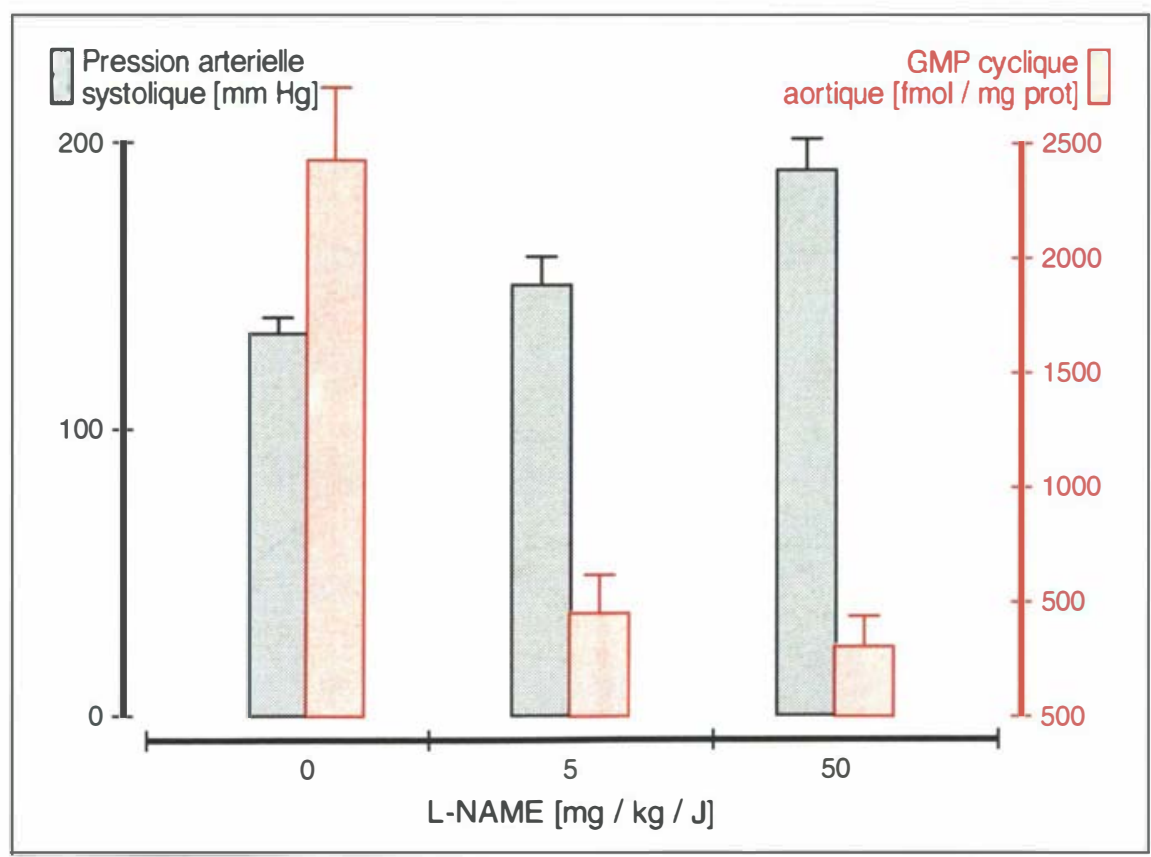

Figure 3. Modèle expérimental d'hypertension artérielle par intoxication chronique à la L-NAME (1 mois). Augmentation dépendante de la dose de la pression et diminution dépendante de la dose du taux de GMP cyclique dans la paroi artérielle [12]. 
gine génétique, et modèles d'hypertensions secondaires, induites expérimentalement.

Le modèle du rat spontanément hypertendu de souche Okamoto (SH) est le plus connu et le plus utilisé. Comme son nom l'indique, cette souche développe spontanément une hypertension artérielle sévère, indépendante des conditions d'environnement. Dans ce modèle, de nombreux arguments militent en faveur d'une anomalie primitive de la fonction de contraction et d'une hypertrophie de la cellule musculaire lisse artérielle. Mais existe-t-il une anomalie conjointe de la vasodilatation dépendante de l'endothélium? Certains auteurs ont rapporté une diminution de la relaxation dépendante de l'endothélium d'anneaux aortiques isolés provenant de rats $\mathrm{SH}[17,18]$. Mais d'autres auteurs ont observé que la fonction de relaxation dépendante de l'endothélium de vaisseaux résistifs provenant de rats $\mathrm{SH}$ est strictement normale [19]. L'utilisation d'analogues de la L-arginine inhibant la NO-synthase permet à nouveau d'évaluer l'état de la voie du NOGMP cyclique dans ce modèle d'hypertension artérielle d'origine génétique. L'administration aiguë de nitro-arginine, antagoniste compétitif de la L-arginine sur la NO-synthase, augmente la pression artérielle de façon identique chez le rat $\mathrm{SH}$ anesthésié et chez la souche témoin normotendue [20]. Dans notre expérience [21], le taux basal de GMP cyclique dans la paroi aortique du rat SH n'est pas différent de celui des témoins normotendus. L'administration chronique de L-NAME augmente la pression artérielle et diminue le taux de GMP cyclique de façon identique chez le rat $\mathrm{SH}$ et chez le rat normotendu. De plus, l'administration chronique de LNAME s'accompagne d'une mortalité importante et précoce $(>50 \%$ après 15 jours d'administration) chez le rat $\mathrm{SH}$. Ces différents arguments ne militent pas en faveur d'une importante anomalie primitive de la fonction de relaxation dépendant du $\mathrm{NO}$ chez le rat $\mathrm{SH}$.

En revanche, lors d'une surcharge chronique en sodium, la vasodilatation dépendante de l'endothélium semble amplifiée. Le modèle DOCA$\mathrm{m} / \mathrm{s} n^{\circ} 10$ vol. 9, octobre 93 sel induit une hypertension artérielle sévère en combinant une augmentation des apports en sel (eau contenant $1 \%$ de $\mathrm{NaCl}$ ) et l'imposition d'un frein puissant à l'excrétion sodée rénale (intoxication aux minéralocorticoïdes plus néphrectomie unilatérale). Dans ce modèle, la fonction de relaxation du vaisseau dépendant de l'endothélium, particulièrement active, semble jouer un rôle de contre-régulation, limitant l'augmentation de pression. De plus, les vaisseaux résistifs ont une plus forte réponse aux agents pharmacologiques vasorelaxants dépendants de l'endothélium [22].

Le rôle de l'activation de la voie arginine-NO-GMP cyclique dans la régulation de l'homéostasie sodée est également suggéré par les expériences de rein isolé perfusé. L'administration aiguë de L-NAME dans cette préparation diminue le flux plasmatique rénal et augmente d'autant plus la pression de perfusion que les animaux ont été pré-traités de façon chronique par un régime riche en sel [16]. Récemment, Schultz et Tolins ont montré qu'à pression de perfusion constante, la L-NMMA diminuait l'excrétion urinaire de sodium in vivo et qu'un prétraitement des animaux par un régime riche en sel augmentait la réponse à la L-NMMA [23].

Le rôle de la voie arginine-NO-GMP cyclique dans la contre-régulation à un apport excessif en sel est également fortement suggéré par la réponse du rat de Dahl à l'administration de L-arginine [24]. Le rat de Dahl sensible au sel est un modèle génétique d'hypertension en interaction avec l'environnement sodé. $\mathrm{La}$ souche de rat, dite sensible au sel, est pratiquement normotendue lorsque le régime sodé est normal, mais la pression artérielle augmente significativement lorsque ces animaux sont soumis à un régime riche en sel. Dans la souche résistante au sel, l'apport excessif de sel ne s'accompagne pas d'une augmentation détectable de la pression artérielle, et l'administration de fortes doses de L-arginine n'a pas d'effet sur la pression artérielle. En revanche, l'administration aiguë d'un antagoniste de l'arginine augmente la pression et diminue la filtration glomérulaire plus dans la souche résis- tante soumise à la surcharge sodée que dans la souche sensible. A l'inverse, l'administration aiguë de fortes doses de L-arginine diminue la pression artérielle dans la souche sensible au sel soumise à une intoxication chronique par le sel, et l'administration chronique de L-arginine prévient le développement de l'hypertension.

L'ensemble de ces données physiopathologiques est en faveur d'un rôle important de cette voie dans l'adaptation à la surcharge sodée. Dans le modèle physiologique de surcharge en sel, l'activation de la voie du NOGMPc semble importante pour le maintien de la pression artérielle à un niveau normal. Dans le modèle DOCA-sel, l'élévation de pression artérielle a lieu malgré l'activation de cette voie. Chez le rat de Dahl sensible au sel, un déficit génétiquement déterminé de cette voie pourrait rendre compte de l'élévation de pression artérielle en présence d'un excès de sel.

Chez l'homme, le rôle de la fonction vasodilatatrice endothéliale dans l'hypertension n'a pu être abordé que par l'étude de l'action loco-régionale d'agents pharmacologiques ayant pour cible l'endothélium. Panza et al. [25] ont rapporté, chez des patients ayant une hypertension essentielle, une diminution de la réponse vasodilatatrice à l'injection intra-artérielle d'acétylcholine, comparée à celle observée chez un groupe témoin normotendu. Cette diminution de sensibilité à l'acétylcholine s'accompagne, chez les malades, d'une diminution de la réponse vasoconstrictrice aux antagonistes de l'arginine [26]. Inversement, la perfusion de L-arginine, si elle n'a pas d'effet sur le tonus vasodilatateur de base chez le témoin normotendu ni chez l'hypertendu, augmente la sensibilité à l'acétylcholine chez le témoin normotendu et ne la modifie pas chez l'hypertendu [27]. Ces études ne permettent pas de conclure sur un éventuel déficit de cette voie dans l'hypertension artérielle essentielle humaine. Néanmoins, elles démontrent le rôle limitant du substrat (Larginine) dans des conditions de forte stimulation chez l'individu normal sain, et des différences notables de réponse aux agents pharmacologiques 
de cette voie chez l'hypertendu : diminution de sensibilité à l'acétylcholine, aux antagonistes de l'arginine et à la L-arginine elle-même. Un effet bénéfique de la perfusion de substrat exogène (L-arginine) a été également rapporté dans l'hypercholestérolémie expérimentale [28] et humaine [29].

\section{Implication de la voie de la NO-synthase dans d'autres situations physiopathologiques cardiovasculaires}

La sécrétion de NO par l'endothélium est sous le contrôle hémodynamique des variations de contraintes de cisaillement, proportionnelles à la vélocité et à la viscosité sanguines, et inversement proportionnelles aux dimensions vasculaires. Toute situation modifiant ces paramètres modifie les contraintes de cisaillement et donc, probablement, la sécrétion de NO. La pression sanguine n'est pas un déterminant direct des contraintes de cisaillement. Elle peut intervenir indirectement dans leur déterminisme en modifiant les dimensions vasculaires selon la relation asymptotique positive pression-volume qui dépend, bien sûr, de la pression, mais également, et surtout, des propriétés viscoélastiques propres à la nature de chaque paroi vasculaire.

Physiologiquement, lors de la vasodilatation périphérique métabolique induite par l'exercice physique (territoire musculaire), la digestion (territoire splanchnique), ou l'augmentation de fréquence cardiaque (territoire coronaire), les artères de conductance adaptent leurs diamètres en réponse à une augmentation du flux sanguin (figure 1). Expérimentalement, une augmentation chronique du flux peut être induite par la création d'une fistule artério-veineuse. L'induction d'un court-circuit artério-veineux se traduit par une augmentation des vélocités sanguines dans le segment artériel situé en amont de la fistule et une éventuelle diminution de vélocité dans le segment d'aval (figure 4). Dans notre expérience [30], le taux de GMP cyclique augmente dans le segment artériel d'amont et diminue dans le segment artériel d'aval, pro-

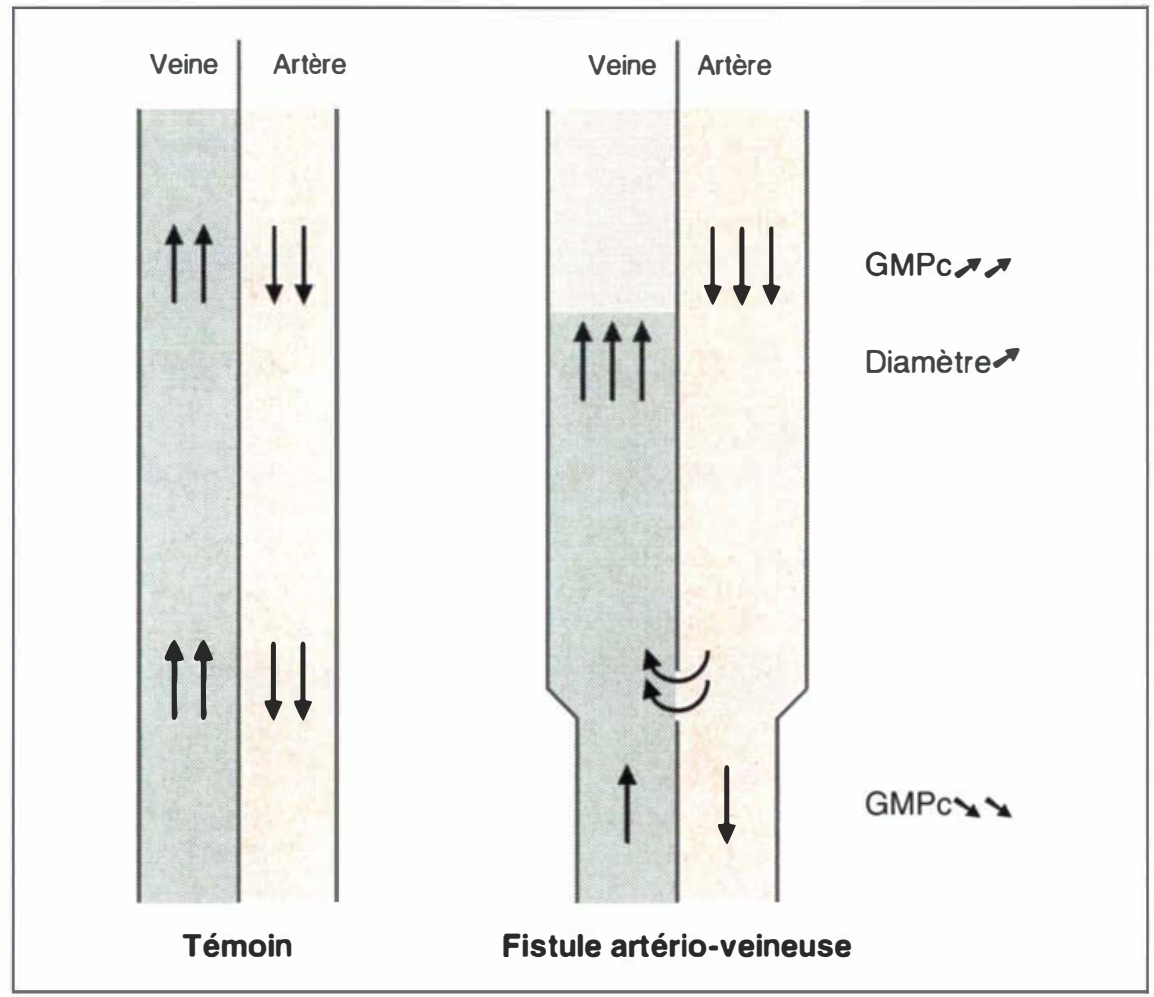

Figure 4. Schématisation de la transduction du " signal de flux " dans une fistule artério-veineuse. L'augmentation du flux entraîne une augmentation de GMP cyclique, une vasodilatation immédiate et une adaptation structurale (développement d'une méga-artère).

tions de contraintes de cisaillement. Inversement, Languille et al. [10] ont montré que la diminution chronique du flux dans une carotide de lapin s'accompagne de modifications structurales de la paroi artérielle (diminution de la lumière vasculaire et de l'épaisseur de la paroi). Cliniquement, la création d'une fistule artério-veineuse radiale pour hémodialyse s'accompagne du développement d'une méga-artère. Les modifications structurales de la paroi artérielle en rapport avec des modifications de flux sont probablement " initiées" par l'endothélium, peut-être via l'activation de la NO-synthase endothéliale [9].

L'insuffisance cardiaque représente une des situations physiopathologiques dans laquelle le flux sanguin est diminué. La diminution du débit cardiaque est alors associée à une élévation des pressions dans la petite circulation (artère pulmonaire) et à une pression artérielle systémique nor- male, voire basse. Dans un modèle d'insuffisance cardiaque provoqué par un infarctus du myocarde chez le rat, Ontkean et al. [31] ont montré une diminution de la relaxation dépendante de l'endothélium d'anneaux isolés d'artère pulmonaire. Des résultats parallèles mais moins puissants étaient obtenus avec des anneaux aortiques. La plus grande sensibilité des anneaux d'artère pulmonaire comparée aux anneaux aortiques pourrait être indirectement liée à la différence des régimes de pression dans la petite et la grande circulation, alors que le débit est diminué de façon identique dans les deux circulations. L'augmentation de pression en amont du ventricule gauche défaillant augmente le rayon de l'artère pulmonaire (relation pression-volume). De ce fait les contraintes de cisaillement diminuent dans l'artère pulmonaire en rapport avec la diminution du débit et l'augmentation du rayon. Inversement, dans les artères en aval du ventricule 
gauche défaillant, la diminution de pression tend à diminuer le rayon artériel. De ce fait, les variations de contraintes de cisaillement ne seraient dues qu'aux variations de flux dans les artères périphériques. Le taux de GMP cyclique aortique tend à diminuer chez les animaux en insuffisance cardiaque, malgré l'augmentation de la concentration plasmatique de facteur atrial natriurétique [32].

Les dimensions des gros vaisseaux artériels se modifient progressivement avec le vieillissement. Le diamètre artériel augmente et la paroi artérielle se rigidifie, en rapport avec des modifications structurales de la matrice extracellulaire. Expérimentalement, la relaxation dépendante de l'endothélium des gros vaisseaux diminue avec l'âge [33]. Dans notre expérience, le taux de GMP cyclique diminue aussi dans la paroi artérielle avec le vieillissement.

$\mathrm{Au}$ total, la fonction endothéliale relaxante, dépendante de la NOsynthase et du GMP cyclique, joue un rôle tonique dans le contrôle de la vasomotricité artérielle basale. L'activité de la NO-synthase endothéliale est contrôlée par le taux de calcium libre intracellulaire. In vitro des substances vasoactives, telles que l'acétylcholine et la bradykinine, ont un effet vasorelaxant par augmentation du calcium libre dans la cellule endothéliale. In vivo, l'activité basale de la NO-synthase endothéliale dépend des contraintes de cisaillement qu'exerce l'écoulement du sang sur l'endothélium. La pression artérielle n'a probablement pas d'effet direct sur cette fonction endothéliale. Elle n'interagit qu'indirectement avec cette fonction, lorsque des modifications de flux ou de calibre sont associées aux variations de pression. A l'inverse le blocage aigu ou chronique de la NO-synthase par des antagonistes compétitifs de la L-arginine s'accompagne d'une augmentation de la pression et d'une diminution des flux. Dans le modèle du rat spontanément hypertendu, la voie Larginine-NO-GMP cyclique paraît normale. L'activation de la voie Larginine-NO-GMP cyclique paraît jouer un rôle important dans l'adaptation à une surcharge en sel. Un défaut de cette contre-régulation pourrait expliquer certaines hyperten$\mathrm{m} / \mathrm{s} n^{\circ} 10 \mathrm{vol}$. 9, octobre 93 sions essentielles dépendantes du sel. Chez l'homme hypertendu, une diminution de sensibilité aux agents pharmacologiques de cette voie a été rapportée, sans que l'on puisse actuellement affirmer l'existence d'une anomalie endothéliale primitive dans l'hypertension essentielle.

Les contraintes de cisaillement dépendent de la vélocité sanguine et des dimensions vasculaires, mais pas directement de la pression. Des situations d'augmentation chronique des vélocités, telles que les fistules artérioveineuses, ou, à l'inverse, de diminution chronique des flux par amputation d'un territoire vasculaire, s'accompagnent de modifications chroniques de la fonction vasodilatatrice endothéliale retentissant sur la structure de la paroi artérielle. L'insuffisance cardiaque est un exemple où la diminution des contraintes de cisaillement pourrait être responsable d'un dysfonctionnement endothélial. L'augmentation des dimensions vasculaires pourrait être responsable de la diminution de la fonction vasodilatatrice endothéliale observée au cours du vieillissement artériel

\section{TIRÉS A PART}

J.B. Michel.

\section{Summary}

Nitrogen monoxyde and arterial hypertension

The relaxing endothelial function, dependent on NO-synthase activity and cyclic GMP production, plays a tonic role in the regulation of arterial vasomotricity. The endothelial NO-synthase activity is dependent on endothelial cell calcium. In vitro, vasoactive substances, such as acetylcholine and bradykinin, exert their relaxing action on smooth muscle cells by increasing calcium in endothelial cells. In vivo, the basal NOsynthase activity is under the control of the shear stress exerted by blood on the endothelial surface. Acute or chronic blockade of NOsynthase activity with L-arginine antagonists is associated with a rise in pressure and a fall in flow. The renal vascular bed is particularly sensitive to NO-synthase blockade. In primary, essential hypertension, mimicked by the spontaneously hypertensive rat, the vasorelaxing $\mathrm{NO}$-synthase activity appears to be normal. In contrast, sodium overload is associated with an overactivity of the NOsynthase. A defect in this counterregulation may participate in some models of essential hypertension depending on salt overload, such as the experimental model of salt sensitive Dahl rat. Shear stress depends on blood flow velocity and on the dimension of the arterial lumen and is not directly dependent on pressure. Pathophysiological situations associated with chronic changes in blood flow, such as arteriovenous fistula, are associated with chronic modifications of shear stress and endothelial relaxing function, leading to modifications of arterial wall structure. Congestive heart failure is an example of a possible chronic decrease in shear stress leading to a decrease in the endothelial relaxing function. The arterial lumen widening could be involved in the diminished endotheliumderived relaxing factor activity observed with aging. 